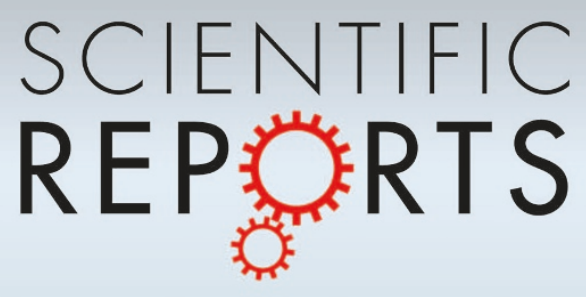

OPEN

SUBJECT AREAS:

ELECTRONIC DEVICES

ELECTRONIC MATERIALS

MATERIALS SCIENCE

CHARACTERIZATION AND

ANALYTICAL

TECHNIQUES

Received

30 September 2013

Accepted

18 March 2014

Published

2 May 2014

Correspondence and requests for materials should be addressed to E.G. (egarf@rutgers. edu)

\section{Oxygen Incorporation in Rubrene Single Crystals}

\author{
Daniel D. T. Mastrogiovanni' ', Jeff Mayer ${ }^{2}$, Alan S. Wan², Aleksey Vishnyakov ${ }^{3}$, Alexander V. Neimark ${ }^{3}$, \\ Vitaly Podzorov ${ }^{4,5}$, Leonard C. Feldman ${ }^{4,5}$ \& Eric Garfunkel ${ }^{1,5}$
}

'Department of Chemistry and Chemical Biology, Rutgers University Piscataway, NJ 08854, ${ }^{2}$ Evans Analytical Group, East Windsor, NJ 08520, ${ }^{3}$ Department of Chemical Engineering, Rutgers University Piscataway, NJ 08854, ${ }^{4}$ Department of Physics, Rutgers University Piscataway, NJ 08854, Institute for Advanced Materials, Devices and Nanotechnology, Rutgers University Piscataway, NJ 08854.

Single crystal rubrene is a model organic electronic material showing high carrier mobility and long exciton lifetime. These properties are detrimentally affected when rubrene is exposed to intense light under ambient conditions for prolonged periods of time, possibly due to oxygen up-take. Using photoelectron, scanning probe and ion-based methods, combined with an isotopic oxygen exposure, we present direct evidence of the light-induced reaction of molecular oxygen with single crystal rubrene. Without a significant exposure to light, there is no reaction of oxygen with rubrene for periods of greater than a year; the crystal's surface (and bulk) morphology and chemical composition remain essentially oxygen-free. Grand canonical Monte Carlo computations show no sorbtion of gases into the bulk of rubrene crystal. A mechanism for photo-induced oxygen inclusion is proposed.

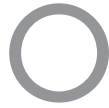

rganic molecular crystals are ideal structures to explore fundamental electronic and optical properties of organic semiconductors (OSCs). Devices comprising single crystals of small molecules eliminate defects associated with grain boundaries or amorphous phases ${ }^{1}$. This facilitates the development of basic fundamental models of charge generation, transport and relaxation in this important class of materials. An understanding of the transport and photo-physics of single crystals enables greater insight into the ultimate behavior obtainable in organic thin-film devices. Such studies form a base-line for the more complex structures likely to appear in applied organic electronics.

Environmental stability may hinder the commercialization of organic electronics. Deleterious effects of atmospheric gases (water, oxygen) are well documented for polymer and small molecule thin-film-based organic photovoltaics (OPVs) ${ }^{2,3}$, light emitting diodes (OLEDs) ${ }^{4}$, sensors ${ }^{5}$, and field effect transistors (OFETs) ${ }^{6,7}$. Broadly speaking, the nature of unwanted effects can be classified as physical, e.g. delamination of a thin-film from an electrode, or chemical, e.g. oxidation of the active component to generate donors/traps for charge carriers. Similar interactions with single crystal OSCs have received much less attention and motivate the present study.

Rubrene is an ideal compound to study the interactions between oxygen and single crystal OSCs for three reasons. First, as single crystal rubrene displays the "best" electronic and optoelectronic properties of any OSC, with charge carrier mobility reaching up to $20 \mathrm{~cm}^{2} \mathrm{~V}^{-1} \mathrm{~s}^{-1}$ in reproducible experiments, it has been the subject of numerous studies ${ }^{8-18}$. Secondly, the existing understanding of the mechanism of reactions between oxygen and free rubrene molecules in gas or solution phases ${ }^{19-22}$, may facilitate the understanding of the interaction of oxygen with a solid-state form of this material, namely single crystalline rubrene. Finally, as intrinsic rubrene $\left(\mathrm{C}_{42} \mathrm{H}_{28}\right)$ does not contain oxygen, effects of adsorption and incorporation can be examined at a sensitive level.

There are conflicting reports in the literature regarding the interaction of oxygen and single crystal rubrene and the accompanying effect on rubrene's electrical and optical properties. (Unless otherwise noted, all further references to rubrene are intended to be understood as single crystal rubrene.) Maliakal et al. observed an increase in conductivity of rubrene crystals after exposure to oxygen at elevated temperatures, which was interpreted as $p$ type doping of rubrene by oxidation ${ }^{23}$. Zhang et al. observed that the apparent mobility of rubrene FETs decreased after exposure to hydrogen gas and attributed this to the chemical reduction of oxidized rubrene ${ }^{24}$, although this interpretation remains controversial given the existence of hydrogen traps ${ }^{25}$.

On the other hand, Chen et al. systematically observed a decrease of the field-effect mobility and conductivity of rubrene OFETs upon photo-oxidation ${ }^{26}$. Krellner et al., using temperature-dependent space-charge-limited current spectroscopy, reported a bulk trap state at $0.27 \mathrm{eV}$ above the valence band generated after intentional 
photo-oxidation ${ }^{27}$. The authors noted that a trap state of lower density was also observed in crystals that had not been deliberately oxidized and was attributed to unintentional oxidation during sample preparation. These effects are also consistent with reports by Karak, et al. ${ }^{28}$. Najafov, et al. used photocurrent excitation spectroscopy to observe a persistent decrease in the dark conductivity and photo-conductivity of rubrene crystals after an initial exposure to visible light in oxygen, followed by an extended incubation in the same environment without illumination ${ }^{29}$. The authors proposed that micrometer-deep diffusion of oxygen into the bulk of the crystal occurring in the dark was triggered by the initial photo-oxidation. Using mass spectroscopy Käfer and Witte observed rubrene peroxide $\left(\mathrm{C}_{42} \mathrm{H}_{28} \mathrm{O}_{2}\right)$ at the surface of poly-crystalline rubrene films after exposure to ambient conditions for a week ${ }^{30}$. Note that the vacuum evaporation used to produce these poly-crystalline films differs greatly from the physical vapor transport (PVT) growth of highquality free standing rubrene single crystals studied here.

Several models may describe the interaction between molecular oxygen and single crystal rubrene. These models represent concepts proposed by ourselves and other aforementioned authors, and focus mainly on the structural relationship between oxygen and rubrene. In the dark, organic single crystals can be viewed as either an impenetrable solid or a semi-porous material into which $\mathrm{O}_{2}$ can diffuse. Under illumination, a number of oxidized rubrene species can be created as the result of photo-oxidation. These species can be found at the surface, but can also exist in the bulk either due to incorporation of oxygen in the bulk during crystal growth or through a mechanism of diffusion into the lattice and a subsequent reaction with rubrene molecules. One can also envision the initiation and progression of the oxidation as either occurring homogeneously along the crystal surface or at specific sites, e.g. molecular step edges or other surface defects as illustrated in Fig 1.

\section{Results}

In the current study, we utilize dynamic secondary ion mass spectroscopy (SIMS), aided by exposing the crystals to isotopically labeled oxygen ${ }^{18} \mathrm{O}_{2}$, x-ray photoelectron spectroscopy (XPS, Supporting Information), and atomic force microscopy (AFM), to reveal the mechanism, the dynamics and the length scale of oxidation and oxygen permeation in the model organic semiconductor, single crystal rubrene. Our results clearly show that there is little oxygen present at the surface or in the bulk of the crystals, even after prolonged storage in oxygen/air, as long as the crystals are kept in the dark. However, concurrent exposure to light and oxygen results in gradual surface oxidation and permeation of oxygen into the crystals at depths up to several hundred nanometers. Aided by AFM imaging, we propose a mechanism whereby oxygen incorporation initiates and proceeds at step edges of the molecular solid surface. This model is consistent with the recent observations of nucleation of selfassembled monolayers along the step edges of rubrene ${ }^{31}$. This implies that molecular step edges on pristine $p$-type molecular crystals are more reactive, and, given standard silanol-based chemistries, possibly oxidized.

Physical vapor transport enables the growth of single crystals of rubrene with high purity and structural order ${ }^{1,32}$. To study the oxygen penetration, SIMS was used to characterize rubrene crystals photooxidized in a controlled manner. Isotopically enriched $(97 \%){ }^{18} \mathrm{O}_{2}$ was used to distinguish between intentional oxidation and oxygen that may have been unintentionally incorporated in the samples during the crystal growth, or handling in the ambient. At atmosphere, samples received at most $1 \mathrm{~h}$ ambient illumination, with more typical exposure times less than $15 \mathrm{~min}$ (see Methods). Briefly, rubrene crystals were loaded into a vacuum chamber fitted with an optical window, and the system was evacuated to $P \approx 1 \times$ $10^{-6}$ Torr. After that, ${ }^{18} \mathrm{O}_{2}(P \approx 350$ Torr $)$ was introduced, and the crystals were illuminated with an external halogen lamp with a broad spectrum (500-1000 nm) for a period of time. The UV component of the lamp is cut off by the glass window of the chamber. The samples were then removed from the chamber and transferred ex-situ for SIMS analysis.

Experiments used large-area $\left(\sim 1.5 \times 1.5 \mathrm{~cm}^{2}\right)$ flat single crystals cleaved into two pieces, thus allowing the elimination of potential variations amongst different crystals. Fig. 2 shows SIMS data obtained on the two pieces of the same rubrene crystal, both exposed to the same oxygen environment. One was illuminated, while the other (control sample) remained in the dark. Profiles of oxygen concentrations for both ${ }^{18} \mathrm{O}$ and ${ }^{16} \mathrm{O}$ as a function of depth into the crystal are displayed in Fig. 2 for both samples.

It is clear that only the illuminated sample exhibits penetration of oxygen into the bulk of the crystal, with ${ }^{18} \mathrm{O}$ concentration decreasing with depth to a level of $10^{17}$ atoms $/ \mathrm{cm}^{3}$ (the detection limit of our instrument) at $\sim 600 \mathrm{~nm}$ below the surface. The concentration of ${ }^{18} \mathrm{O}$ near the surface of the illuminated crystal is $\sim 4 \times 10^{20} \mathrm{~cm}^{-3}$, which is still considerably smaller than the concentration of $\mathrm{O}$ atoms expected for a monolayer of rubrene peroxide, $\sim 2.8 \times 10^{21} \mathrm{~cm}^{-3}$. It is important to note that SIMS was used for elemental analysis in the results presented here. We have separately performed TOF-SIMS experiments which provide information on the molecular species that are sputtered from the surface, and we obsesrve rubrene with one and two oxygen atoms incorporated in the sputtered molecular fragments. Note that crystalline rubrene has 4 molecules per unit cell with a volume of $2800 \AA^{3}$ (for details of the crystal structure, see ref. 33).

The crystal that was kept in the dark exhibits no additional ${ }^{18} \mathrm{O}$ in the bulk. The near-surface region of both the illuminated and control samples exhibits a moderate increase in ${ }^{18} \mathrm{O}$ and ${ }^{16} \mathrm{O}$, which is likely due to a combination of physisorbed molecular oxygen and rubrene
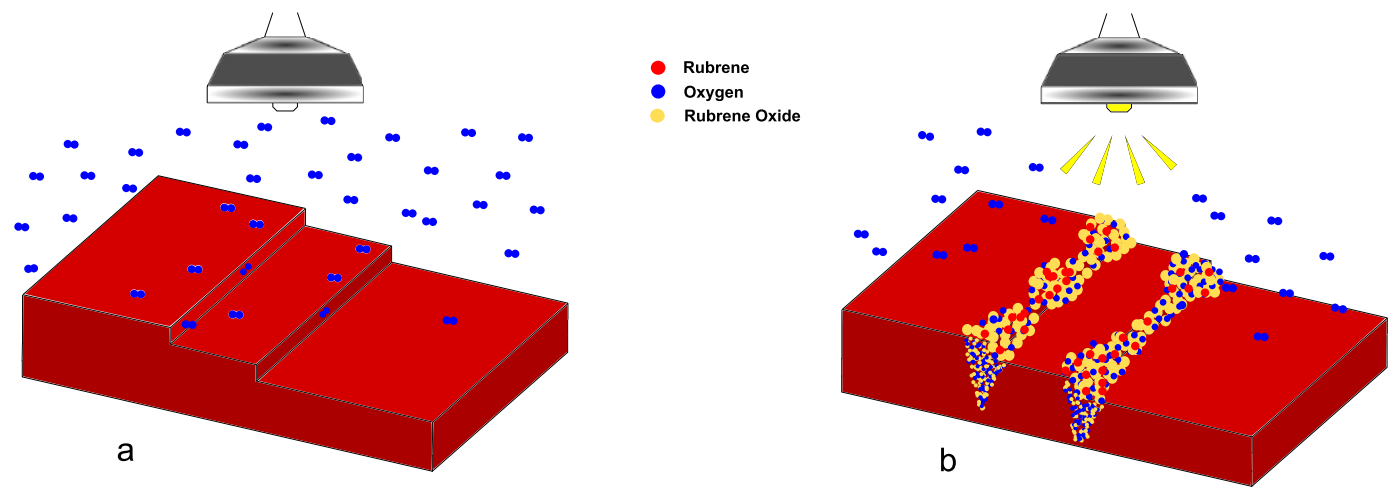

Figure $1 \mid$ Schematic illustration of the proposed interaction of $\mathrm{O}_{2}$ with single crystal rubrene. In the absence of light, oxygen is not able to oxidize densely packed terraces or penetrate in the crystal bulk. Under illumination the oxygen reacts with rubrene at steps/surface defects, opening up the crystal lattice to allow for penetration into the bulk. 

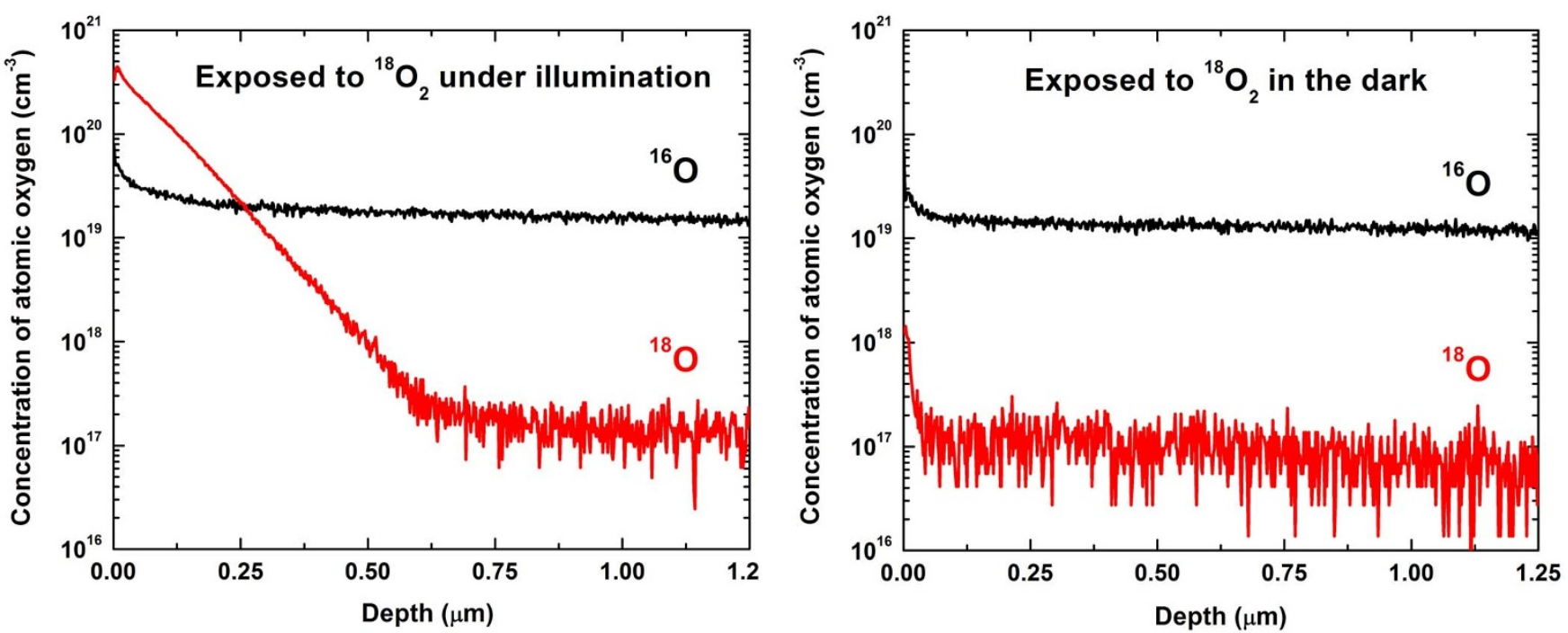

Figure $2 \mid$ Concentration of atomic oxygen as a function of depth into the crystal revealed by SIMS. A comparison is given between the oxygen isotope $\left({ }^{18} \mathrm{O}\right)$ and naturally occurring oxygen $\left({ }^{16} \mathrm{O}\right)$ background. For this experiment, a large monolithic single crystal was carefully cut into two halves that were simulateously exposed to isotopically enriched molecular oxygen $\left({ }^{18} \mathrm{O}_{2}\right)$, one piece - in the dark (right panel), and the other piece - under illumination (left panel).

oxide formed during the sample handling. In either case, the difficulty in precisely interpreting SIMS data within the first few nanometers of the surface is well-known. Nevertheless, it is clear from the SIMS data (Fig. 2, right panel) that the oxygen concentration at the surface of the crystal kept in the dark $\left(\sim 1.5 \times 10^{19} \mathrm{~cm}^{-3}\right.$ for ${ }^{16} \mathrm{O}$, and $\sim 10^{18} \mathrm{~cm}^{-3}$ for ${ }^{18} \mathrm{O}$ ) is orders of magnitude smaller than the concentration corresponding to a fully oxidized surface $\left(3 \times 10^{21} \mathrm{~cm}^{-3}\right)$, thus suggesting that only approximately one out of two hundred rubrene molecules at the surface of pristine single crystals is oxidized. The low level of oxygen incorporation in the absense of light is further corroberated by XPS studies (see Supporting Information).

The SIMS experiments (here and below) clearly show that the concurrent exposure to light and oxygen is necessary for a surface oxidation and permeation of oxygen into rubrene crystals. Thus, a model for this up-take emerges that views the crystal as impenetrable to $\mathrm{O}_{2}$ in the absence of photo-excitation (Fig. 1, left), with illumination initiating an oxidation of the surface defects and facilitating a pathway for oxygen incorporation.

Fig. 3 provides a comparison of SIMS profiles obtained from three crystals exposed to various combinations of oxygen/illumination to further confirm the model discussed above. ${ }^{18} \mathrm{O}$ is found at a depth of $\sim 50 \mathrm{~nm}$ and $\sim 150 \mathrm{~nm}$ in the crystals exposed to ${ }^{18} \mathrm{O}_{2}$ and light for 6 and 24 hours, respectively (red and black curves). This is close to the factor of two expected in a simple diffusion model enabled by light exposure. The third rubrene crystal was first exposed to ${ }^{18} \mathrm{O}_{2}$ for $18 \mathrm{~h}$ in the dark and then for an additional $6 \mathrm{~h}$ under illumination (blue
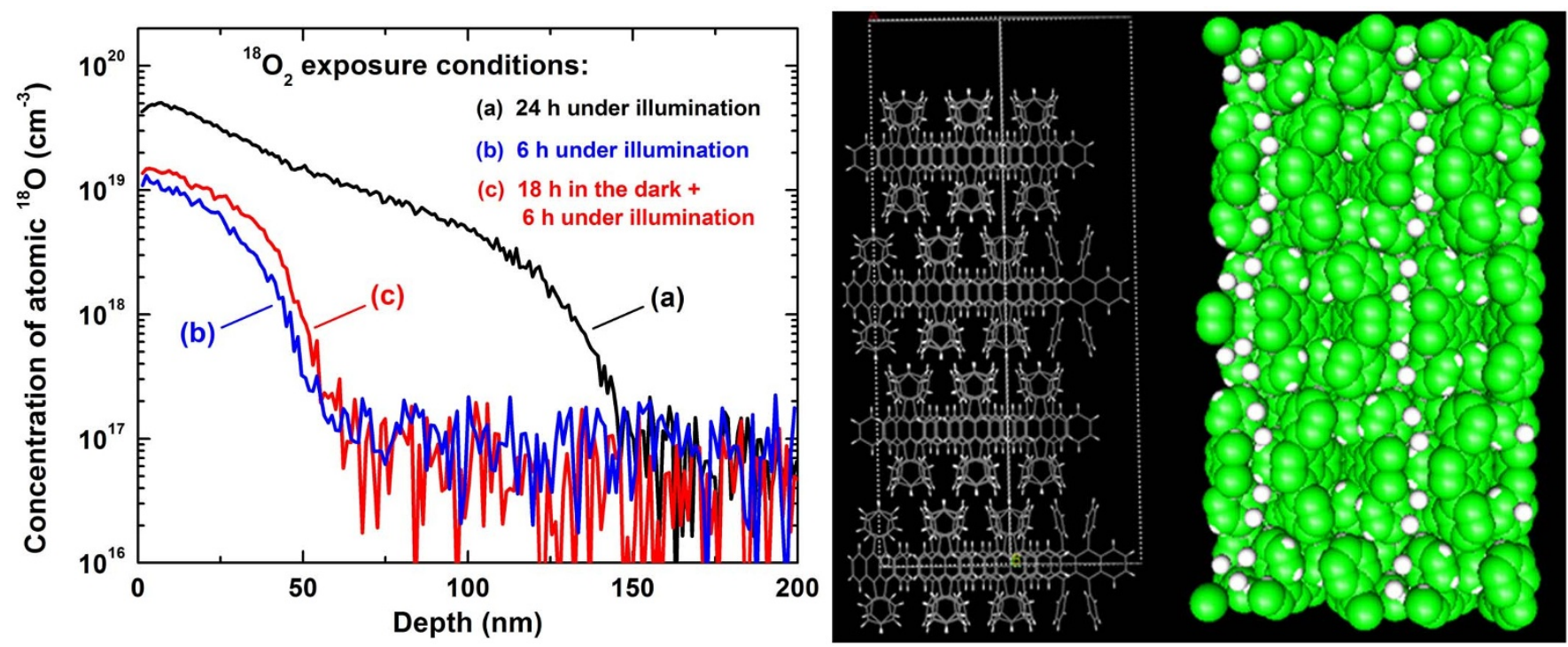

Figure 3 (Left) Depth profiles of oxygen concentration obtained by SIMS in rubrene crystals subjected to various photo-oxidation treatments. (a) Crystal exposed to ${ }^{18} \mathrm{O}_{2}$ and illumination for $24 \mathrm{~h}$; (b) crystal exposed to ${ }^{18} \mathrm{O}_{2}$ and illumination for $6 \mathrm{~h}$; (c) crystal exposed to ${ }^{18} \mathrm{O}_{2}$ for $18 \mathrm{~h}$ in the dark and then for additional $6 \mathrm{~h}$ under illumination. The dependence with respect to light exposure, combined with exposure in the dark, provide evidence that penetration and diffusion of oxygen does not occur in the dark. Oxygen incorporation requires both oxygen exposure and light illumination. (Right) Wire-frame and space-filled models of single crystal rubrene are shown from the perspective of the $\boldsymbol{b}$ - $\boldsymbol{c}$ planes. The green and white balls represent carbon and hydrogen, respectively. Based on our calculations, we find that single crystal rubrene cannot absorb oxygen into its bulk without having a significant lattice distortion. 
curve): the SIMS concentration profile for this sample closely follows the "6 hours + light" profile (red curve). This composite further proves that exposure to oxygen in the dark is insufficient for oxygen up-take, but that exposure combined with illumination is required for oxygen incorporation. Such impenetrability of single crystalline rubrene to $\mathrm{O}_{2}$ is in agreement with grand canonical Monte Carlo simulations indicating that rubrene shows no intrinsic porosity towards $\mathrm{O}_{2}$ or even He (Fig. 3 and Supporting Information).

Having established that oxygen can react with the surface and enters rubrene bulk only in the presence of light, it is of interest to understand the mechanism of oxygen penetration. AFM imaging of the $(\mathbf{a}, \boldsymbol{b})$ facet of rubrene crystals stored in air in the dark reveals that the surface prior to photo-oxidation consists of flat terraces vertically separated by $\sim 1.5 \mathrm{~nm}$-tall molecular steps, which is consistent with previous studies (Fig. 4 , top $)^{34,35}$. When illumination is minimized, no changes in the morphology of the surface are observed for periods extending up to a year. However, photo-oxidation initiates a roughening primarily along the step edges (Fig. 4, bottom); this image corresponds to a $12 \mathrm{~h}$ exposure to ${ }^{18} \mathrm{O}_{2}$ under our typical illumination. The AFM profile of the photo-oxidized rubrene reveals a height increase of $\sim 1 \mathrm{~nm}$ continuous along the step edges. The step edges also exhibit isolated larger features, the "beads", on the order of 7$10 \mathrm{~nm}$ in height as also observed by Thompson et al. ${ }^{36}$ The remainder of the rubrene terrace remains relatively flat and largely unaffected. Although having a full three dimensional nanoscale image of the oxygen density would help clarify the structure and give clues as to how oxygen is distributed in the film, this is not yet technically feasible for this class of materials. Atom probe tomography and TEM result in excessive damage to such samples. Current NanoSIMS instruments are limited to $50 \mathrm{~nm}$ lateral resolution at best, and are not optimzed for depth profiling. SEM or SAM would likewise not yield a meaningful nanoscale 3D map of low level oxygen impurities in an organic crystal.

The photo-oxidation of rubrene is strongly temperature dependent. There is a substantial increase in the amount of ${ }^{18} \mathrm{O}$ present both on and below the surface, as the oxidation temperature is increased (Fig. 5). Possible causes include: (i) an increase in the rate of the oxygen - rubrene reaction (activated reaction between $\mathrm{O}_{2}$ and rubrene molecules); (ii) an increased rate of surface diffusion, step flow, and formation of surface defects (e.g., an increase in step edge roughening, generation of rubrene "holes" or isolated rubrene molecules at the terraces); or (iii) an increase in the rate of $\mathrm{O}_{2}$ diffusion in the bulk (once the rubrene lattice is distorted around the oxidized surface molecules). A control rubrene crystal heated at $85^{\circ} \mathrm{C}$ for $24 \mathrm{~h}$ in the dark retains the terraced structure of pristine rubrene crystals (similar to the one shown in the top panel of Fig. 4). Contrary to this control sample, heating the surface under illumination changes the structure drastically (Fig. S3).

Finally, we note that there is a uniform ${ }^{16} \mathrm{O}$ background in the rubrene bulk with an easily detectable atomic concentration of about $1.5 \times 10^{19} \mathrm{~cm}^{-3}$. (As explained in the Supplementary Information the temperature dependence of this "background oxygen level" indicates that it exceeds the instrumental background of the SIMS apparatus). Given the concentration of rubrene molecules in the crystal is $1.43 \times 10^{21} \mathrm{~cm}^{-3}$, this level of oxygen represents approximately one rubrene peroxide molecule per 200 rubrene molecules in the bulk of the crystal. This background level extends at least several microns into the bulk of the crystal, possibly throughout the entire crystal.
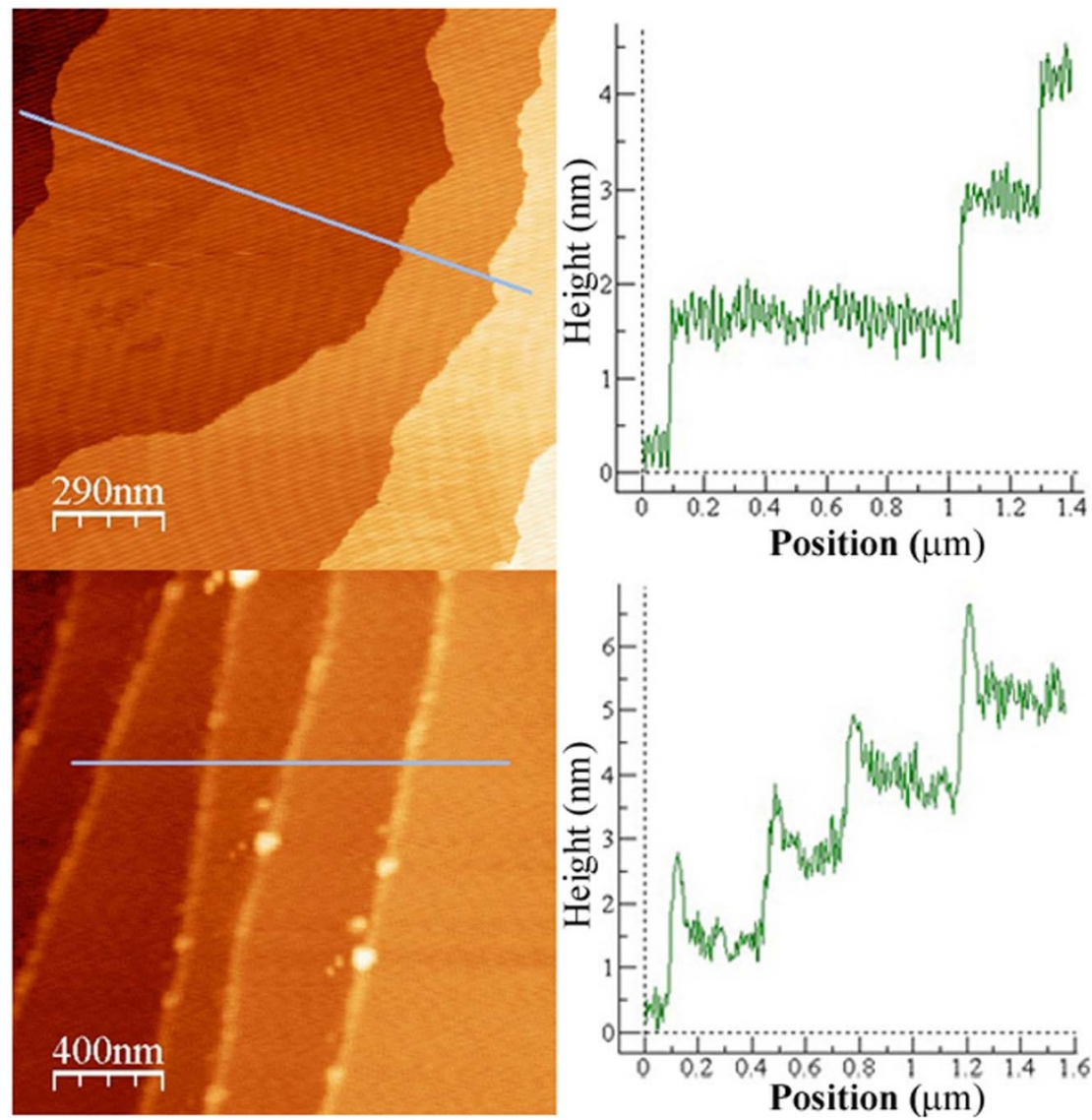

Figure $4 \mid$ AFM of rubrene crystal before and after photo-oxidation. Pristine rubrene crystals exhibits well-resolved molecular terraces at the (a,b)-facet with a height of $1.5 \mathrm{~nm}$ (top). This is the case even for the crystals stored in air for years, as long as exposure to visible light is brief. Illumination in the presence of oxygen results in a preferential modification of molecular steps (bottom). The height scale is $7 \mathrm{~nm}$ (top) and $13 \mathrm{~nm}$ (bottom). This suggests that photo-induced interaction of rubrene with oxygen occurs (at least initially) primarily at molecular step edges and other surface defects. 


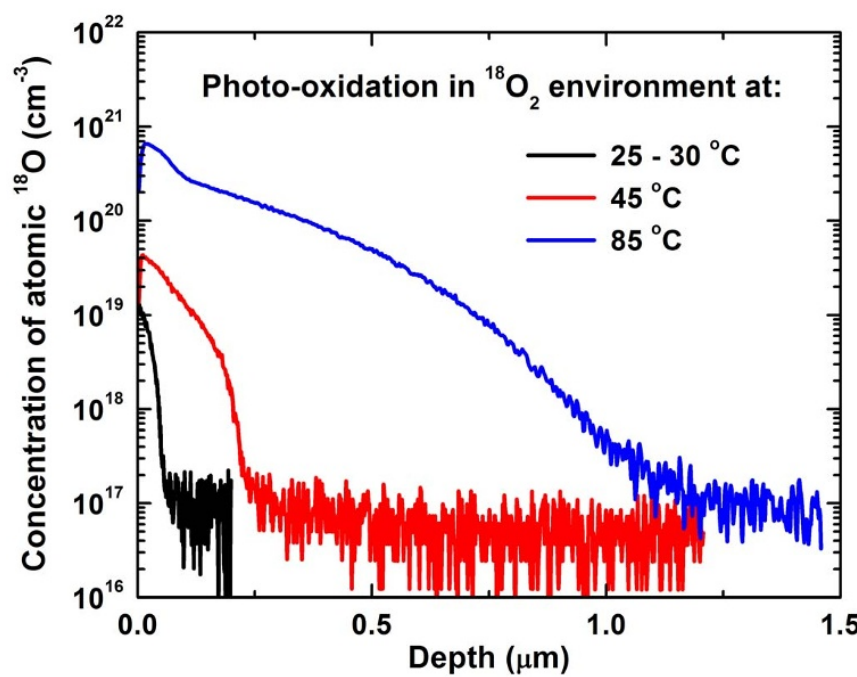

Figure $5 \mid$ Photo-oxidation of single crystal rubrene in ${ }^{18} \mathrm{O}_{2}$ performed at different temperatures (indicated). The SIMS profiles show that both the penetration depth and concentration of ${ }^{18} \mathrm{O}$ increase significantly with temperature. Annealing of the crystals in oxygen atmosphere in the dark at similar temperatures does not lead to such a pronounced enhancement of oxygen incorporation.

The exact source and chemical nature of this oxygen are unclear and require further investigation.

\section{Discussion}

With these data, one can propose a model of how oxygen interacts with rubrene single crystals. In the dark, $\mathrm{O}_{2}$ exhibits only minimal interaction with the rubrene surface and is unable to penetrate into the bulk. Illumination of rubrene with light of sufficient energy (above the rubrene band-gap) in the presence of oxygen leads to the gradual oxidation of rubrene. Oxidation of the rubrene tetracene backbone has been shown both experimentally and theoretically to be more favorable than that of the pendant phenyl groups, and thus accelerated reaction at this location is expected ${ }^{37,38}$. Given the structure of the single crystal unit cell, it is at the step edge that the acene backbone of the rubrene molecule is most exposed. In some cases, as with the formation of rubrene endoperoxide, oxidation of the acene will lead to a loss of planarity in the backbone ${ }^{30}$. This could lead to a distortion of the local crystal lattice that ultimately results in the roughening observed at the step edges. However, this selective roughening of the step edge (on the order of $\sim 1 \mathrm{~nm}$, covering a small fraction of the surface) cannot account for the oxygen detected by SIMS hundreds of nanometers into the bulk. Thus, a probable mechanism that accounts for all these observations is a gradual permeation of $\mathrm{O}_{2}$ into the crystal under photo-excitation with visible light that occurs through defects and a subsequent chemical reaction between $\mathrm{O}_{2}$ and rubrene. Though the details of this process require further study, we suggest that incorporation of oxygen proceeds via a stepby-step process. In this process, rubrene molecules along the step edges are first photo-oxidized, which distorts the surrounding crystalline lattice sufficiently, so that $\mathrm{O}_{2}$ can diffuse in further through the oxidized regions to reach rubrene previously protected by the densely packed crystal structure (Fig. 1, right).

\section{Methods}

Single crystals of rubrene were prepared via the physical vapor transport growth method with He (Matheson, UHP 99.995\%) as the carrier gas. Starting material consisted of as-received powder (Sigma Aldrich, sublimed grade) and/or purified crystals (typically recrystallized 2-4 times). All crystals used for this study were of the "platelet" morphology (area $\geq 1 \mathrm{~cm}^{2}$, thickness $\geq 0.5 \mathrm{~mm}$ ). Crystals were mounted on a metal foil using silver paint prior to oxidation and analysis. The base pressure of the oxidation chamber was $1 \times 10^{-6}$ Torr. Illumination intensity in photo-oxidation experiments was $250 \pm 25 \mathrm{~mW} / \mathrm{cm}^{2}$ and the resulting temperature at the sample was below $30^{\circ} \mathrm{C}$ as measured in-situ by thermocouple. After oxidation samples were transferred ex-situ for analysis.

SIMS data were acquired using a PHI ADEPT 1010 Quadrapole analyzer instrument. Secondary ions were detected in the negative ion mode, employing $\mathrm{Cs}^{+}$as the primary ion source. The sputter depths are accurate to approx. 1\% (calibrated by stylus profilometry of the sputtered crater). The size of the crater is $500 \times 500 \mathrm{um}$, and the area of acceptance is $10 \%$ of the total sputtered area. Charge compensation was employed using electron bombardment.

XPS spectra were taken with a Perkin-Elmer hemispherical analyzer with a nonmonochromatic $\mathrm{Al} \mathrm{K} \alpha \mathrm{x}$-ray source $\mathrm{hv}=1486.6 \mathrm{eV}$. At $17.9 \mathrm{eV}$ pass energy, the full width at half maximum (FWHM) of the $\mathrm{Cu} 2 \mathrm{p} 3 / 2$ core level is $1.2 \mathrm{eV}$. AFM images were obtained using a Digital Instruments Multimode AFM with a Nanoscope IV controller operating in "tapping mode."

1. Podzorov, V. Organic single crystals: Addressing the fundamentals of organic electronics. MRS Bull. 38, 15-24 (2013).

2. Tavakkoli, M. et al. Progress in stability of organic solar cells exposed to air. Sol. Energy Mater. Sol. Cells 95, 1964-1969 (2011).

3. Norrman, K., Gevorgyan, S. A. \& Krebs, F. C. Water-Induced Degredation of Polymer Solar Cells Studied by $\mathrm{H}_{2}{ }^{18} \mathrm{O}$ Labeling. ACS Appl. Mater. \& Interfaces $\mathbf{1}$, 102-112 (2009).

4. Levermore, P. A. et al. Phosphorescent organic light-emitting diodes for highefficacy long-lifetime solid-state lighting. J. Photon. Energy. 2, 021205-1-8 (2012).

5. Angione, M. D. et al. Carbon based materials for bio-sensing. Mater. Today 14, 424-433 (2011)

6. Petrovic, A. \& Bratina, G. Interface resistivity and lifetime of thin film transistors exposed to ambient air. Appl. Phys. Lett. 94, 123301-1-3 (2004).

7. Glowacki, E. D. et al. Organic Electronics: Hydrogen-Bonded Semiconducting Pigments for Air-Stable Field-Effect Transistors. Adv. Mater. 25, 1563-1569 (2013).

8. Podzorov, V. et al. Intrinsic charge transport on the surface of organic semiconductors. Phys. Rev. Lett. 93, 086602-1-4 (2004).

9. Okada, Y., Sakai, K., Uemura, T., Nakazawa, Y. \& Takeya, J. Charge transport and hall effect in rubrene single-crystal transistors under high pressure. Phys. Rev. B 84, 245308-1-4 (2011).

10. Najafov, H., Lee, B., Zhou, Q., Feldman, L. \& Podzorov, V. Observation of longrange exciton diffusion in highly ordered organic semiconductors. Nat. Mater. 9 , 938-943 (2010)

11. Xie, W. et al. High-Mobility Transistors Based on Single Crystals of Isotopically Substituted Rubrene- $\mathrm{d}_{28}$. J. Phys. Chem. C 117, 11522-11529 (2013).

12. Dacuña, J., Xie, W. \& Salleo, A. Estimation of the spatial distribution of traps using space-charge-limited current measurements in an organic single crystal. Phys. Rev. B 86, 115202-1-6 (2012).

13. Briseno, A. L. et al. High-Performance Organic Single-Crystal Transistors on Flexible Substrates. Adv. Mater. 18, 2320-2324 (2006).

14. da Silva Filho, D. A., Kim, E. G. \& Brédas, J. L. Transport Properties in the Rubrene Crystal: Electronic Coupling and Vibrational Reorganization Energy. Adv. Mater. 17, 1072-1076 (2005).

15. Hulea, I. N. et al. Tunable Frölich polarons in organic single-crystal transistors. Nat. Mater. 5, 982-986 (2006).

16. Reese, C. \& Bao, Z. High-Resolution Measurement of the Anisotropy of Charge Transport iin Single Crystals. Adv. Mater. 19, 4535-4538 (2007).

17. Troisi, A. Prediction of the Absolute Charge Mobility of Molecular Semiconductors: the Case of Rubrene. Adv. Mater. 19, 2000-2004 (2007).

18. Reyes-Martinez, R. A., Ramasubramaniam, A., Briseno, A. L. \& Crosby, A. J. The Intrinsic Mechanical Properties of Rubrene Single Crystals. Adv. Mater. 24, 5548-5552 (2012).

19. Stevens, B. \& Algar, B. E. Photoperoxidation of unsaturated organic molecules. II. Autoperoxidation of aromatic hydrocarbons. J. Phys. Chem. 72, 3468-3474 (1968).

20. Usui, Y., Shimizu, N. \& Mori, S. Mechanism on the Efficient Formation of Singlet Oxygen by Energy Transfer from Excited Singlet and Triplet States of Aromatic Hydrocarbons. B. Chem. Soc. Jpn. 65, 897-902 (1992).

21. Wilson, T. Excited Singlet Molecular Oxygen in Photooxidation. J. Am. Chem. Soc. 88, 2898-2902 (1966).

22. Wu, K. C. \& Trozzolo, A. M. Production of singlet molecular oxygen from the oxygen quenching of the lowest excited singlet state of rubrene. J. Phys. Chem. 83, 2823-2826 (1979).

23. Maliakal, A. et al. Mechanism for Oxygen-Enhanced Photoconductivity in Rubrene: Electron Transfer Doping. Chem. Mater. 21, 5519-5526 (2009).

24. Zhang, K. et al. Control of charge mobility in single-crystal rubrene through surface chemistry. Org. Electron. 11, 1928-1934 (2010).

25. Northrup, J. E. \& Chabinyc, M. L. Gap states in organic semiconductors: Hydrogen- and oxygen-induced states in pentacene. Phys. Rev. B 68, 041202-1-4.

26. Chen, Y. \& Podzorov, V. Bias Stress Effect in "Air-Gap" Organic Field-Effect Transistors. Adv. Mater. 24, 2679-2684 (2012).

27. Krellner, C. et al. Density of bulk trap states in organic semiconductor crystals: Discrete levels induced by oxygen in rubrene. Phys. Rev. B 75, 245115-1-5 (2007). 
28. Karak, S., Lim, J. A., Ferdous, S., Duzhko, V. V. \& Briseno, A. L. Photovoltaic Effect at the Schottky Interface with Organic Single Crystal Rubrene. Adv. Funct. Mater. 24, 1039-1046 (2014).

29. Najafov, H., Mastrogiovanni, D., Garfunkel, E., Feldman, L. C. \& Podzorov V. Photon-assisted oxygen diffusion and oxygen related traps in organic semiconductors. Adv. Mater. 23, 981-985 (2011).

30. Käfer, D. \& Witte, G. Growth of crystalline rubrene films with enhanced stability. Phys. Chem. Chem. Phys. 7, 2850-2853 (2005).

31. Lee, B., Choi, T.-J., Cheong, S.-W. \& Podzorov, V. Nanoscale Conducting Channels at the Surface of Organic Semiconductors Formed by Decoration of Molecular Steps with Self-Assembled Molecules. Adv. Func. Mater. 19, 3726-3730 (2009).

32. Kloc, C., Simpkins, P. G., Siegrist, T. \& Laudise, R. Physical vapor growth of centimeter-sized crystals of $\alpha$-hexathiophene. J. of Cryst. Growth 182, 416-427 (1997).

33. Jurchescu, O. D., Meetsma, A. \& Palstra, T. T. M. Low-temperature structure of rubrene single crystals grown by vapor transport. Acta Crystall. B 62, 330-334 (2006).

34. Menard, E., Marchenko, A., Podzorov, V., Gershenson, M., Fichou, D. \& Rogers, J. Nanoscale surface morphology and rectifying behavior of a bulk single-crystal organic semiconductor. Adv. Mater. 18,1552-1556 (2006).

35. Fumagalli, E. et al., Oxidation Dynamics of Epitaxial Rubrene Ultrathin Films. Chem. Mater. 23, 3246-3253 (2011).

36. Thompson, R. J. et al. Evolution of the surface morphology of rubrene under ambient conditions. Appl. Phys. Lett. 98, 053302-1-053302-3 (2011).

37. Tsetseris, L. \& Pantelides, S.-T. Large impurity effects in rubrene crystals: Firstprinciples calculations. Phys. Rev. B 78, 115205-1-115205-5 (2008).

38. Tsetseris, L. \& Pantelides, S.-T. Modification of the electronic properties of rubrene crystals by water and oxygen-related species. Org. Electron. 10, 333-340 (2009).

\section{Acknowledgments}

The authors would like to thank Q. Zou for use of the AFM, and S. Shubeita for discussion. This work was finacncially supported by NSF grant No. DMR-1006740.

\section{Author Contributions}

V.P. helped initiate the work based on earlier studies in his lab. D.D.T.M., E.G. and L.C.F. designed the experiments. D.D.T.M. performed most of the experiments, and provided AFM and XPS analysis. A.S.W. and J.M. performed the SIMS analysis. A.V.N. and A.V. carried out Monte Carlo simulations. The first draft of the paper was provided by D.D.T.M. with E.G., L.C.F. and V.P. contributing extensively to the final manuscript.

\section{Additional information}

Supplementary information accompanies this paper at http://www.nature.com/ scientificreports

Competing financial interests: The authors declare no competing financial interests.

How to cite this article: Mastrogiovanni, D.D.T. et al. Oxygen Incorporation in Rubrene Single Crystals. Sci. Rep. 4, 4753; DOI:10.1038/srep04753 (2014).

This work is licensed under a Creative Commons Attribution-NonCommercialShareAlike 3.0 Unported License. The images in this article are included in the article's Creative Commons license, unless indicated otherwise in the image credit; if the image is not included under the Creative Commons license, users will need to obtain permission from the license holder in order to reproduce the image. To view a copy of this license, visit http://creativecommons.org/licenses/by-nc-sa/3.0/ 